\title{
Europäisierung des Bank- und Wertpapierhandelsstrafrechts
}

\section{Einleitung}

Der deutsche Gesetzgeber hatte in den letzten drei Jahren gleich mehrere europäische Rechtsvorschriften in deutsches Recht umzusetzen ${ }^{1}$. Dies ist rechtstechnisch gesehen keine leichte Aufgabe. Denn der deutsche Gesetzgeber hatte hierbei die europäische Sicht- und Vorgehensweise des europäischen Bank- und Wertpapierhandelsrechts in die vollständig andere deutsche Gesetzes-Systematik zu überführen. Um so mehr ist zu begrüßen, dass sich der deutsche Gesetzgeber mit Akribie dieser rechtstechnisch anspruchsvollen Anforderung widmete, indem er europäische Regelungen gleich in so zahlreiche und unterschiedliche deutsche Gesetze - wie das aktuelle Wertpapierhandelsgesetz (WpHG), das Strafgesetzbuch (StGB), das Börsengesetz (BörsG) sowie die Marktmanipulations-Konkretisierungs-Verordnung (MaKonV) - überführte.

Die Erfüllung dieser Aufgabe ist unverzichtbar, bildet doch die Harmonisierung nationalen Rechts die Grundlage des Entstehen eines einheitlichen, europäischen Banken- und Wertpapiermarktes. Andererseits birgt die Umgestaltung des deutschen Rechts nach europäischen Vorgaben, speziell im deutschen Bank- und Wertpapierhandelsstrafrecht erhebliche Gefahren: so kann die Integration europäischer Normen in das fest gefügte deutsche System des Bank und Wertpapierrecht das nationale (deutsche)Recht erheblich verkomplizieren. Speziell unter strafrechtlichem Aspekt stellt sich die Frage, ob eine derartige Rechtslage noch dem grundgesetzlichen Gebot der konkreten Bestimmung eines Straftatbestandes ${ }^{2}$ genügt.

In diesem Beitrag soll über die derzeit überaus komplexe Rechtslage am Beispiel der Straf- und Bußgeldnormen des aktuellen deutschen Bank- und Wertpapierhandelsrechts informiert werden. Dem deutschen Gesetzgeber sei bereits an dieser Stelle ins Auftragsbuch geschrieben, dass es mit der bloßen Übernahme europäischer Regelungen in das komplexe System des deutschen Rechts, speziell im Bank und Wertpapierhandelsrecht, nicht getan ist. Hier bedarf es einer ausreichend durchdachten Neustrukturierung und systematischen Zusammenfassung der bestehenden deutschen Bankund Wertpapierhandelsgesetze, mit ihren nahezu unzähligen Einzelvorschriften, in ein neues Bank- und Kapitalmarkt-Gesetz.

1 Zum europäischen Bankrecht im Einzelnen siehe: Krimphove: Europäisches Bankrecht, Eine ökonomisch/historische Betrachtung der Reaktion des Europäischen Gesetzgebers, in: Klein, Pieper, Ress (Hrsg.): Gedächtnisschrift für Albert Bleckmann, Köln 2007, (Veröffentlichung im August 2007).

2 Siehe $\S 1$ StGB und der in ihm enthaltene Grundsatz: nulla poena sine lege. 


\section{Die Europäisierung des deutschen Bank- und Wertpapierrechts}

Fast völlig unbeachtet von der öffentlichen Meinung nahm der deutsche Gesetzgeber mit der Umsetzung so bedeutender europäischer Regelungswerke wie die der

- Richtlinie 2003/6/EG des Europäischen Parlaments und des Rates vom 28. Januar 2003 über die Insider-Geschäfte und Marktmanipulationen (MarktmissbrauchsRichtlinie) (ABl. Nr. L 96 vom 14.04.2003, S. 16 ff.)

- Richtlinie 2003/71/EG des Europäischen Parlamentes und des Rates vom 4. November 2003 betreffend den Prospekt, der beim öffentlichen Angebot von Wertpapieren oder bei deren Zulassung zum Handel zu veröffentlichen ist, und zur Änderung der Richtlinie 2001/34/EG (ABl. Nr. L 345 vom 31.12.2003, S. 64 ff.);

- Richtlinie 2003/124/EG der Kommission vom 22. Dezember 2003 zur Durchführung der Richtlinie 2003/6/EG des Europäischen Parlamentes und des Rates betreffend die Begriffsbestimmung und die Veröffentlichung von Insider-Informationen und die Begriffsbestimmung der Marktmanipulation (ABl. Nr. L 339 vom 24.12.2003, S. 70 ff.)

- Richtlinie 2003/125/EG der Kommission vom 22. Dezember 2003 zur Durchführung der Richtlinie 2003/6/EG des Europäischen Parlamentes und des Rates in Bezug auf sachgerechte Darbietung von Anlageempfehlungen und die Offenlegung von Interessenkonflikte(ABl. Nr. L 339 vom 24.12.2003, S. 73 ff.)

- Richtlinie 2004/25/EG des Europäischen Parlaments und des Rates vom 21. April 2004 betreffend Übernahmeangebote (ABl. Nr. L 142 vom 30. 4.2004, S. 12 ff)

- Richtlinie 2004/39/EG vom 21. April 2004 über Märkte für Finanzinstrumente, zur Änderung der Richtlinien 85/611/EWG und 93/6/EWG des Rates und der Richtlinie 2000/12/EG des Europäischen Parlaments und des Rates und zur Aufhebung der Richtlinie 93/22/EWG des Rates (ABl. Nr. L 145 vom 30.04.2004, S. 1 ff.)

- Richtlinie 2004/72/EG der Kommission vom, 29. April 2004 zur Durchführung der Richtlinie 2003/6/EG des Europäischen Parlaments und des Rates vom 28. Januar 2003 über die Insider-Geschäfte und Marktmanipulationen (MarktmissbrauchsRichtlinie) (ABl. Nr. L 162 vom 30.04.2004, S. 70 ff.)

- Richtlinie 2005/60/EG des Europäischen Parlaments und des Rates vom 26.Oktober 2005 zur Verhinderung der Nutzung des Finanzsystems zum Zwecke der Geldwäsche und der Terrorismusfinanzierung (AB1. Nr. L 309 vom 25.11.2005, S. 15 ff.)

- sowie die

- Verordnung 2273/2003/EG der Kommission vom 22. Dezember 2003 zur Durchführung der Richtlinie 2003/6/EG des Europäischen Parlaments und des Rates vom 28. Januar 2003 über die Insider-Geschäfte und Marktmanipulationen (ABl. Nr. L 336 vom 23.12.2003, S. 33 ff.)

- Verordnung 809/2004/EG der Kommission vom 29. April 2004 zur Umsetzung der Richtlinie 2003/71/EG betreffend die in Prospekten enthaltene Informationen sowie das Format, die Aufnahme von Information mittels Verweis auf die Veröffentlichung solcher Prospekte und die Verbreitung von Werbung (ABl. Nr. L 215 vom 16.06.2004, S. 3 ff.) 
massiven Einfluss auf die Sanktionstatbestände wichtiger Bank- und Wertpapierhandelsgesetze. Er griff damit erheblich in das Strafrecht (betreffend der Anleger, Banken, Wertpapierhändler aber auch der Anlageberater und Vermögensverwalter) ein.

Der inhaltliche Eingriff in das nationale Recht aller europäischen Mitgliedstaaten ist zwingend erforderlich, will man europaweit einheitliche Bedingungen für Bankgeschäfte schaffen und damit einen einheitlichen europäischen Banken- und Wertpapiermarkt etablieren. Mit der europäischen Rechtsangleichung geht ebenfalls die gemeinschaftsweite Vereinheitlichung und Stärkung des Anlegerschutzes, die Erleichterungen der Banken- und Wertpapierhandelsaufsicht, sowie letztlich die Erweiterung der Austauschmöglichkeiten von grenzüberschreitenden Bankdienstleistungen und produkten einher.

Der deutsche Gesetzgeber hat sich allerdings speziell im Bereich des Banken- und Wertpapierrechtstrafrechts schwer getan: Statt einer - zugegebenermaßen überaus anspruchsvollen - kompletten systematischen Einarbeitung europäischer Vorgaben in das System des deutschen Rechts, scheint der deutsche Gesetzgeber europarechtliche Vorgaben in der Weise in das deutsche Recht einzufügen, dass er die europäischen Regeln lediglich zu dem Bestand des deutschen Rechts »addiert«. Allenfalls gleicht er dann offensichtliche, inhaltliche Unstimmigkeiten in Einzelfällen durch eine »Konkretisierung einzelner Rechtsbegriffe « wieder aus. Als Folge dieser Gesetzgebungspraxis herrscht heute unter Anlegern, Banken, Wertpapierhändlern, Anlageberatern und Vermögensverwaltern Unklarheit sowohl über den Inhalt des deutschen Bank- und Wertpapierstrafrechts als auch seiner Verbote und Sanktionen.

Diese, seit Jahren zu beobachtende, legislative Vorgehensweise findet ihre Erklärung in dem Zusammentreffen der europäischen Normen auf die differenziert entwickelte, deutsche Rechtsdogmatik mit ihrer stringenten Gesetzessystematik. Die deutsche, streng durchdachte Rechts-Struktur existiert nicht nur innerhalb eines jeden einzelnen deutschen Gesetzes, z.B. dem Börsengesetz, dem Kreditwesengesetz oder dem Wertpapierhandelsgesetz. Sie findet sich - bzw. fand sich - auch gesetzesübergreifend in der Gesamtanlage des deutschen Bank- und Wertpapierhandelsrechts. Andere Rechtsordnungen tun sich zugegebenermaßen mit der Umsetzung europäischer Vorgaben nicht so schwer. Denn sie verfügen in einem weitaus geringeren Maße über eine stringente Rechtssystematik ${ }^{3}$.

Die rein additive Übernahme europäischen Rechts in die historisch gewachsene deutsche Gesetzessystematik erspart dem deutschen Gesetzgeber zwar das dogmatisch mühevolle Durchdringen der vielschichtigen Systematik und z.T. ihrerseits schwerverständlichen Regelungsinhalte des deutschen Bank- und Wertpapierrechts. Diese gesetzgeberische Vorgehensweise hat allerdings den Nachteil, den Umfang des deutschen Rechts um zahlreiche Inhalte lediglich quantitativ zu vergrößern, seine Inhalte demgegenüber immer abgesonderter, beziehungsloser und unzusammenhängender darzustellen und somit seine Anwendung in der Praxis immer undurchschaubarer und undurchdringlicher zu machen.

3 Siehe Krimphove: Europäisches Sachenrecht, Lohmar 2006. 
Diese Tendenz der Rechtsetzung ist auch in anderen Rechtsgebieten zu beklagen. Für die zahlreichen strafrechtlichen Änderungen und europäisch bedingten Modifikationen des deutschen Bank- und Wertpapierhandelsstrafrecht hat dies jedoch die negative Folge, dass das so entstehende Recht nicht mehr strafrechtlichen Grundsätzen genügt: Zweifel ergeben sich hier insbesondere an der Klarheit und Bestimmbarkeit des Straftatbestandes. Undeutlich bleibt auch, wie Aufsichtsbehörden, und Strafverfolgungsbehörden den Inhalt dieses Strafrechts generalpräventiv durchsetzen sollen, wenn dieser nicht nur einem juristischen Laien, sondern auch Fachleuten weitgehend unverständlich ist.

Oben angesprochene Ungereimtheiten zeigen sich im gesamten System des deutschen Bank- und Wertpapierhandelsstrafrechts:

\section{Kapitalanlagebetrug}

Im Bereich des sogen. klassischen Strafrechts des Strafgesetzbuches führten die oben angegebenen europäischen Rechtsvorschriften ${ }^{4}$ bislang zu keiner Änderung deren Wortlautes. Dennoch beeinflussen die europäischen Regelungen die Interpretation auslegungsbedürftiger strafrechtlicher Tatbestandsmerkmale. Als Beispiel mag hier $\S$ 264 a StGB (Kapitalanlagebetrug) angeführt werden:

Strafrechtlich schützt $§ 264$ a StGB den Anleger vor Irreführungen. Im Gegensatz zu $\S 263$ StGB geht Paragraph 264a StGB von einem viel eingeschränkteren Begriff der Irreführung aus: Der Gegenstand der Täuschung in $§ 264 \mathrm{a}$ StGB ist lediglich die Verbreitung unrichtiger »vorteilhafter« oder das Verschweigen »negativer« Angaben über den »Vermögensstand «.

Dieser Irrtum ist auch nur strafrechtlich relevant, wenn er für die Anlegerentscheidung »erhebliche« Umstände betrifft.

Was mit dem Wort »erheblich« gemeint ist bleibt - insbesondere nach der Umsetzung der o.g. europäischer Regelungswerke ${ }^{5}-$ bis heute offen $^{6}$.

4 Siehe Kapitel I.

5 Insbesondere der Richtlinie 2003/6/EG des Europäischen Parlaments und des Rates vom 28. Januar 2003 über die Insider-Geschäfte und Marktmanipulationen (MarktmissbrauchsRichtlinie) (ABl. Nr. L 96 vom 14.04.2003, S. 16 ff.); Richtlinie 2003/124/EG der Kommission vom 22. Dezember 2003 zur Durchführung der Richtlinie 2003/6/EG des Europäischen Parlamentes und des Rates betreffend die Begriffsbestimmung und die Veröffentlichung von Insider-Informationen und die Begriffsbestimmung der Marktmanipulation (ABl. Nr. L 339 vom 24.12.2003, S. 70 ff.); Richtlinie 2003/125/EG der Kommission vom 22. Dezember 2003 zur Durchführung der Richtlinie 2003/6/EG des Europäischen Parlamentes und des Rates in Bezug auf sachgerechte Darbietung von Anlageempfehlungen und die Offenlegung von Interessenkonflikte(ABl. Nr. L 339 vom 24.12.2003, S. 73 ff.) sowie der Verordnung 809/2004/EG der Kommission vom 29. April 2004 zur Umsetzung der Richtlinie 2003/71/EG betreffend die in Prospekten enthaltene Informationen sowie das Format, die Aufnahme von Information mittels Verweis auf die Veröffentlichung solcher Prospekte und die Verbreitung von Werbung (ABl. Nr. L 215 vom 16.06.2004, S. 3 ff.).

6 Auch: Lampe: Der Kreditbetrug, München 1980, S. 49. 


\section{a) Das Merkmal der »Erheblichkeit« aus europarechtlicher Sicht}

Unklar ist insbesondere, ob es auf eine objektive Erheblichkeit der Anlage-Umstände ankommt, oder ob - insbesondere nach der Umsetzung der o.g. Europäischen Richtlinien - die Erheblichkeit der Umstände aus Sicht des Anlegers zu beurteilen ist:

Legt man zur Beantwortung dieser Frage die auf die Richtlinie 2003/124/EG ${ }^{7}$ sowie die Verordnung 2273/2003/EG ${ }^{8}$ zurückgehende Begriffsbestimmung des $§ 2$ der Verordnung zur Konkretisierung des Verbotes der Marktmanipulation (Marktmanipulations-Konkretisierungsverordnung) v. 1. 3. $2005^{9}$ zur »bewertungserheblichen Umständen« zugrunde, kommt es zur Annahme einer bewertungs-erheblichen Tatsache entscheidend auf die subjektive Sichtweise des jeweiligen Anlegers an ${ }^{10}$. Bei dieser Rechtslage hinge die Strafbarkeit von der individuellen und subjektiven Einschätzung des Anlegers bezüglich der Erheblichkeit der Information ab. Dieses Ergebnis erscheint rechtspolitisch überaus zweifelhaft. Denn ausschließlich bei Antragsdelikten kommt der Effekt zum Tragen, die Strafverfolgung von dem Zutun des Geschädigten abhängig zu machen. Während jedoch Antragsdelikte einen eigenen Grund zur Aussetzung der Strafverfolgung bieten, schlösse das Abstellen auf die obige »europäische geprägte « Sichtweise - also auf die individuelle Beurteilung des Anlegers - ohne erkennbaren Grund - nicht erst die Strafverfolgung sondern bereits die Strafbarkeit aus.

Allerdings ist es bislang weder der Rechtsliteratur noch der deutschen Rechtsprechung überzeugend gelungen, das Tatbestandsmerkmal der »Erheblichkeit« in $§ 264 \mathrm{a}$ StGB eindeutig und daher strafrechtskonform zu fassen: Während die h.M. in der Rechtsprechung zur Feststellung der Erheblichkeit daher auf so »blumig, nichtssagende Formulierungen« wie den »verständigen, durchschnittlich vorsichtigen Anleger $\ll$ abstellt $^{11}$, entwickelt die Literatur zu diesem Zweck zahlreiche, sich aber z.T. widersprechende Einzelkriterien ${ }^{12}$. Den wohl weitesten Weg der Auslegung beschreitet Park. Park möchte derartige Auslegungsschwierigkeiten dadurch umgehen, dass er das Merkmal der »Erheblichkeit « in § 264a StGB auf die Anlageentscheidung selbst - und nicht auf die ihr zugrunde liegenden (erheblichen) Umstände - bezieht. Eine solche

7 Richtlinie 2003/124/EG der Kommission vom 22. Dezember 2003 zur Durchführung der Richtlinie 2003/6/EG des Europäischen Parlamentes und des Rates betreffend die Begriffsbestimmung und die Veröffentlichung von Insider-Informationen und die Begriffsbestimmung der Marktmanipulation (ABl. Nr. L 339 vom 24.12.2003, S. 70 ff.).

8 Verordnung 2273/2003/EG der Kommission vom 22. Dezember 2003 zur Durchführung der Richtlinie 2003/6/EG des Europäischen Parlaments und des Rates vom 28. Januar 2003 über die Insider-Geschäfte und Marktmanipulationen (ABl. Nr. L 336 vom 23.12.2003, S. $33 \mathrm{ff}$.)

9 BGBl. I S. 515.

$10 \S 2$ Abs. 1 MaKonV: Bewertungserhebliche Umstände i.S.d. § 20a Abs. 1 Satz1, Nr. 1 des Wertpapierhandelsgesetzes sind Tatsachen und Werturteile, die ein verständiger Anleger bei seiner Anlageentscheidung berücksichtigen würde.

11 Insbes. BGH St. in: Wistra 2005, S. 304 ff., 306 (m.w.H.); Joecks, in: Handbuch des Wirtschaftsstrafrechts, Heidelberg 2004, § 264a StGB, Rn. 46 (m.w.H.).

12 Einzelheiten bei: Park, in: Park (Hrsg.) Kapitalmarktstrafrecht, 1. Aufl. Baden-Baden 2004, $\S 264 \mathrm{a}$ StGB, Rn. 11; Joecks, in: Handbuch des Wirtschaftsstrafrechts, Heidelberg 2004, § 264a StGB, Rn. 46 (m.w.H.). 
Sicht ist dem Wortlaut des $§ 264$ a StGB gerade nicht zu entnehmen ${ }^{13}$. Park selbst gerät dann auch in seiner weiteren Argumentation in Abgrenzungs- und Verständnisschwierigkeiten $^{14}$.

Diese Rechtssituation muss alarmieren, denn weder die Rechtswissenschaft noch die Rechtsprechung arbeiten heute mit einem, den Anforderungen des $\S 1$ StGB genügenden, festumrissenen Tatbestandsmerkmal der »Erheblichkeit «.

Auch der auf der europäischen Richtlinie 2003/6/EG des Europäischen Parlaments und des Rates vom 28. Januar 2003 über die Insider-Geschäfte und Marktmanipulationen (Marktmissbrauchs-Richtlinie) ${ }^{15}$ beruhende $\S 2$ der deutschen Verordnung zur Konkretisierung des Verbots der Marktmanipulation ${ }^{16}$ (im Folgenden MaKonV) kann nicht zur Klärung der »Erheblichkeit« herangezogen werden. Zwar beschreibt § 2 MaKonV den Begriff der »bewertungserheblichen Umstände«. Eine Übernahme dieses Verständnisses auf das der »Erheblichkeit« in $\S 264$ a StGB ist dennoch aus folgenden Gründen ausgeschlossen: Neben dem strafrechtlichen Analogieverbot des $\S 1$ StGB und des Art. 103 GG, bestehen schon grundsätzliche Bedenken, ob eine seitens der Exekutive erlassene Norm - wie die der Marktmanipulations-KonkretisierungsVerordnung - strafbegründend wirken sollen. Dies erscheint verfassungsrechtlich zwar grundsätzlich möglich ${ }^{17}$. Besondere Schwierigkeiten ergeben sich allerdings auch aus der Ableitung dieser Rechtssicht aus dem Recht der Europäischen Gemeinschaften; hier insbesondere aus der Richtlinie 2003/6/EG ${ }^{18}$. Die Richtlinie 2003/6/EG, die der deutsche Gesetzgeber umzusetzen hatte, besitzt nämlich keinen strafgesetzli-

13 Park, in: Park (Hrsg.) Kapitalmarktstrafrecht, 1. Aufl. Baden-Baden 2004, § 264a StGB, Rn. 13.

14 Dreher: Kommentar zum StGB 52 Aufl., § 264a, Rn. 16 (m.w.H.); Cramer/Perron, in: Schönke/Schröder Kommentar zum StGB 27. Aufl. § 264a, Rn. 30 ff. (m.w.H.).

15 ABl. Nr. L 96 vom 14.04.2003, S. 16 ff.); siehe auch: Richtlinie 2003/124/EG der Kommission vom 22. Dezember 2003 zur Durchführung der Richtlinie 2003/6/EG des Europäischen Parlamentes und des Rates betreffend die Begriffsbestimmung und die Veröffentlichung von Insider-Informationen und die Begriffsbestimmung der Marktmanipulation (ABl. Nr. L 339 vom 24.12.2003, S. 70 ff.); Richtlinie 2003/125/EG der Kommission vom 22. Dezember 2003 zur Durchführung der Richtlinie 2003/6/EG des Europäischen Parlamentes und des Rates in Bezug auf sachgerechte Darbietung von Anlageempfehlungen und die Offenlegung von Interessenkonflikte (ABl. Nr. L 339 vom 24.12.2003, S. 73 ff.).

16 Sogen. Marktmanipulations-Konkretisierungs-Verordnung, v. 1 1.3 .2005

17 BVerfGE 14, S. 174 ff, 185; 14, S. 2456 ff., 251; 75, S. 329 ff., 342 (jeweils m.w.H.).

18 Richtlinie 2003/6/EG des Europäischen Parlaments und des Rates vom 28. Januar 2003 über die Insider-Geschäfte und Marktmanipulationen (Marktmissbrauchs-Richtlinie), in: ABl. Nr. L 96 vom 14.04.2003, S. 16 ff.); siehe auch: Richtlinie 2003/124/EG der Kommission vom 22. Dezember 2003 zur Durchführung der Richtlinie 2003/6/EG des Europäischen Parlamentes und des Rates betreffend die Begriffsbestimmung und die Veröffentlichung von Insider-Informationen und die Begriffsbestimmung der Marktmanipulation (AB1. Nr. L 339 vom 24.12.2003, S. 70 ff.); Richtlinie 2003/125/EG der Kommission vom 22. Dezember 2003 zur Durchführung der Richtlinie 2003/6/EG des Europäischen Parlamentes und des Rates in Bezug auf sachgerechte Darbietung von Anlageempfehlungen und die Offenlegung von Interessenkonflikte (ABl. Nr. L 339 vom 24.12.2003, S. 73 ff.) 
chen Charakter. Zum Erlass bzw. zur Beeinflussung nationalen Strafrechts, fehlt dem Europäischen Gesetzgeber aber die Kompetenz ${ }^{19}$. Die Verwendung der Richtlinie 2003/6/EG zum Zweck der Interpretation nationaler strafrechtlich relevanter Begriffe, würde daher dieser Richtlinie für das deutsche Recht einen strafrechtsrelevanten Inhalt zusprechen. Somit würde sie über den Zweck, den Inhalt der Richtlinie 2003/6/EG und der Kompetenz des europäischen Gesetzgebers unzulässig hinausgehen.

\section{b) Unrechtsbewertung und Strafrahmen}

Eine unvollkommene, d.h. eine nicht-systemgerechte, Einbeziehung europäischer Vorgaben in das deutsche Bank- und Wertpapierhandels lässt sich ebenfalls anhand des Vergleiches der Tatbestandsmerkmale und insbesondere der Strafandrohungen von § 263 StGB und § 264a StGB ablesen: Reicht zur Strafbarkeit wegen Betruges i.S.d. § 263 StGB jede Form der Täuschungshandlung (mündlich, schriftlich, konkludent etc.) aus, so verlangt die Täuschungshandlung i.S.d. Kapitalanlagebetruges § 264a StGB eine besonders qualifizierte, schriftliche Darstellungs-Täuschung, nämlich die Irreführung durch Prospekte, Darstellungen oder Übersichten. Überdies genügt nach § 264a StGB die Irreführung eines Einzelnen nicht. Die Kapitalanlagetäuschung erfordert vielmehr, dass sich die oben bezeichnete Täuschungshandlung an »einen größeren Kreis von Personen« richtet.

Erstaunlich oder nahezu widersprüchlich ist, dass der deutsche Gesetzgeber die Qualifizierung des Kapitalanlagebetruges ( $\$ 264 \mathrm{a} \mathrm{StGB}$ ) einem weit geringeren Strafrahmen, als den allgemeinen Betrug ( $\$ 263$ StGB) unterstellt. Hier hätte man annehmen sollen, dass eine (Massen-)Täuschung einer unbestimmten Vielzahl von Anlegern i.S.d. § 264a StGB durch speziell auszuarbeitende Prospekte, Übersichten und Darstellungen nicht nur gefährlicher als die allgemeine Betrugshandlung i.S.d. § 263 StGB ist, sondern auch vom Täter größere kriminelle Energie abverlangt. Diesen Strafgrund des Kapitalanlagebetruges ( $\$ 264 \mathrm{a} \mathrm{StGB}$ ) greift der deutsche Gesetzgeber in seiner Strafrahmenfestlegung jedoch gerade nicht auf.

\section{Verleiten zu Börsenspekulationsgeschäften}

Die Richtlinie 2003/6/EG des Europäischen Parlaments und des Rates vom 28. Januar 2003 über die Insider-Geschäfte und Marktmanipulationen (Marktmissbrauchs-Richtlinie $)^{20}$ ist auch maßgeblich für die Etablierung des Straftatbestandes des Verleiten zu Börsenspekulationsgeschäften $\S \S 61$ i.V.m. § 23 Abs. 1 BörsG geworden. Nach $\S \S 61$ i.V.m. § 23 Abs. 1 BörsG macht sie jeder strafbar, der gewerbsmäßig einen anderen, durch Ausnutzung dessen Unerfahrenheit, zum Abschluss von Börsenspekulationsgeschäften (i.S.d. § 23 Abs. 2 BörsG) oder der Beteiligung an ihnen verleitet.

Obschon speziell die oben genannte europäische Regelung die Einführung dieses Verbotes in allen europäischen Mitgliedstaaten herbeigeführt hat, ist es bedauerlicherweise dem deutschen Gesetzgeber bis heute nicht gelungen, den Begriff des »Verlei-

19 Siehe auch: Schröder: Europäische Richtlinie und deutsches Strafrecht; München 2002, S. 339 ff, 445 ff. (m.w.H.).

20 ABl. Nr. L 96 vom 14.04.2003, S. 16 ff. 
tens $^{21}$ « in $\S 23$ BörsG eindeutig inhaltlich festzulegen ${ }^{22}$. Das Deutungsspektrum reicht von einer bloßen »Verursachung « des Börsenspekulationsgeschäftes ${ }^{23}$ bis hin zu einem »böswilligen Ausnutzen der Entscheidungsunfähigkeit des Anlegers $\ll{ }^{24}$.

Auch die oben erwähnte ${ }^{25}$ europäische Marktmissbrauchs-Richtlinie ${ }^{26}$ und ihre Folgedokumenten $^{27}$ gewähren keine Möglichkeit zu einer Deutung dieses unbestimmten Tatbestandsmerkmals: Zwar führt die Marktmissbrauchs-Richtlinie den Begriff des »Verleitens« in ihrem Art. 3 auf. Weder sie, noch die oben erwähnten Folgedokumente definieren ihn. Zudem verwendet die europäische Marktmissbrauchs-Richtlinie den Begriff des Verleitens nur im Bezug auf Insidergeschäfte, so dass eine Übernahme des in der Marktmissbrauchs-Richtlinie erwähnten Begriffs des »Verleitens « in den Kontext des $§ 264$ a StGB ohnehin zweifelhaft erscheint ${ }^{28}$.

\section{Das Recht der Insiderhandelsverbote}

Überaus komplex überträgt der deutsche Gesetzgeber die zum europäischen Insiderrecht ergangenen Regelungswerke ${ }^{29}$ und deren Sanktionen in sein nationales Insider-Recht.

21 Zum problematischen Merkmal des »Verleitens « im deutschen Strafrecht siehe auch: $\S \S 120,160,323 \mathrm{~b}, 367$ StGB hier aber mit weitaus auslegungsfähigerem Inhalt.

22 siehe auch: Nack: in: Müller-Gugenberger/Bieneck; Wirtschaftsstrafrecht, 4. Aufl. Heidelberg 2006, § 23 BörsG (m.w.H.); Achenbach/Ransierk (Herg.) Handbuch Wirtschaftsstrafrecht: Heidelberg 2004, Kapitel X, Rn 250.

23 BGH NStZ - RR 2002, S. 84 ff. (m.w.H.).

24 so BGH NStZ 2000, S. 36 ff. (m.w.H.).

25 siehe oben: Kapitel I.1.a (m.w.H.).

26 ABl. Nr. L 96 vom 14.04.2003, S. 16 ff.

27 Richtlinie 2003/124/EG der Kommission vom 22. Dezember 2003 zur Durchführung der Richtlinie 2003/6/EG des Europäischen Parlamentes und des Rates betreffend die Begriffsbestimmung und die Veröffentlichung von Insider-Informationen und die Begriffsbestimmung der Marktmanipulation (ABl. Nr. L 339 vom 24.12.2003, S. 70 ff.); Richtlinie 2004/ 72/EG der Kommission vom, 29. April 2004 zur Durchführung der Richtlinie 2003/6/EG des Europäischen Parlaments und des Rates vom 28. Januar 2003 über die InsiderGeschäfte und Marktmanipulationen (Marktmissbrauchs-Richtlinie) (ABl. Nr. L 162 vom 30.04.2004, S. 70 ff.); Verordnung 2273/2003/EG der Kommission vom 22. Dezember 2003 zur Durchführung der Richtlinie 2003/6/EG des Europäischen Parlaments und des Rates vom 28. Januar 2003 über die Insider-Geschäfte und Marktmanipulationen (AB1. Nr. L 336 vom 23.12.2003, S. 33 ff.).

28 Zum Merkmal des Verleitens zu Insider-Geschäften siehe unten: Kapitel I.4. (m.w.H.).

29 Richtlinie 2004/72/EG der Kommission vom, 29. April 2004 zur Durchführung der Richtlinie 2003/6/EG des Europäischen Parlaments und des Rates vom 28. Januar 2003 über die Insider-Geschäfte und Marktmanipulationen (Marktmissbrauchs-Richtlinie) (ABl. Nr. L 162 vom 30.04.2004, S. 70 ff .).

Verordnung (EG) Nr. 2273/2003 der Kommission vom 22. Dezember 2003 zur Durchführung der Richtlinie 2003/6/EG des Europäischen Parlaments und des Rates vom 28. Januar 2003 über die Insider-Geschäfte und Marktmanipulationen (ABl. Nr. L 336 vom 23.12.2003, S. 33 ff.),

Richtlinie 2003/124/EG der Kommission vom 22. Dezember 2003 zur Durchführung der Richtlinie 2003/6/EG des Europäischen Parlamentes und des Rates betreffend die Begriffsbestimmung und die Veröffentlichung von Insider-Informationen und die Begriffsbestimmung der Marktmanipulation (ABl. Nr. L 339 vom 24.12.2003, S. 70 ff.), 
Im Vergleich zu der vormaligen Rechtslage, führt er im Jahr 2005 - aufgrund europäischer Vorgaben ${ }^{30}$ - überaus komplizierte Änderungen ein. In der aktuellen Rechtslage differenziert der deutsche Gesetzgeber inhaltlich und dogmatisch zwischen dem Verbot von Insiderhandlungen, deren Strafbarkeit und zugleich deren Ordnungswidrigkeit.

a) Die allgemeine Regel des Insiderhandelsverbotes

Das Verbot, Insiderinformationen (i.S.d. $§ 13 \mathrm{WpHG)} \mathrm{weiterzugeben} \mathrm{und/oder} \mathrm{zu} \mathrm{nut-}$ zen enthält $\S 14$ WpHG. Dabei stellt $\S 14$ i.V.m. $\S 13$ Abs. 2 WpHG reine Beratungsund Bewertungstätigkeit, ohne die Weitergabe von nichtöffentlichen Informationen von dem Insiderverbot frei.

Abbildung 1: Insider-Handels-Verbote $§ 14 \mathrm{WpHG}$

\begin{tabular}{|l|l|}
\hline Norm & Handlungs-Verbote (i.S.d. § 14 WpHG) \\
\hline 14 Abs. 1 WpHG & $\begin{array}{l}\text { Erwerb oder Veräußerung unter Verwendung der Insiderin- } \\
\text { formation }\end{array}$ \\
\hline 14 Abs. 2 WpHG & $\begin{array}{l}\text { Unbefugtes Mitteilen und Zugänglichmachen von Insiderin- } \\
\text { formation }\end{array}$ \\
\hline 14 Abs. 3 WpHG & $\begin{array}{l}\text { Empfehlung und Verleitung eines anderen - auf Grundlage } \\
\text { der Insiderinformation - zum Erwerb oder Veräußerung von } \\
\text { Insiderpapieren }\end{array}$ \\
\hline
\end{tabular}

Richtlinie 2003/6/EG des Europäischen Parlaments und des Rates vom 28. Januar 2003 über die Insider-Geschäfte und Marktmanipulationen (Marktmissbrauchs-Richtlinie) (AB1. Nr. L 96 vom 14.04.2003, S. 16 ff.)

Richtlinie 89/592/EWG des Rates vom 13. November 1989 zur Harmonisierung der Vorschriften betreffend Insider-Geschäfte (ABl. Nr. L 334 vom 18.11.1989, S. 30 ff.).

30 siehe insbesondere:

Verordnung (EG) Nr. 2273/2003 der Kommission vom 22. Dezember 2003 zur Durchführung der Richtlinie 2003/6/EG des Europäischen Parlaments und des Rates vom 28. Januar 2003 über die Insider-Geschäfte und Marktmanipulationen (ABl. Nr. L 336 vom 23.12.2003, S. 33 ff.),

Richtlinie 2003/6/EG des Europäischen Parlaments und des Rates vom 28. Januar 2003 über die Insider-Geschäfte und Marktmanipulationen (Marktmissbrauchs-Richtlinie) (ABl. Nr. L 96 vom 14.04.2003, S. 16 ff.)

Richtlinie 2003/124/EG der Kommission vom 22. Dezember 2003 zur Durchführung der Richtlinie 2003/6/EG des Europäischen Parlamentes und des Rates betreffend die Begriffsbestimmung und die Veröffentlichung von Insider-Informationen und die Begriffsbestimmung der Marktmanipulation (ABl. Nr. L 339 vom 24.12.2003, S. 70 ff.),

Richtlinie 2004/72/EG der Kommission vom, 29. April 2004 zur Durchführung der Richtlinie 2003/6/EG des Europäischen Parlaments und des Rates vom 28. Januar 2003 über die InsiderGeschäfte und Marktmanipulationen (Marktmissbrauchs-Richtlinie) (ABl. Nr. L 162 vom 30.04.2004, S. 70 ff .). 
Das Insiderhandelsverbot des $\S 14 \mathrm{WpHG}$ unterscheidet heute nicht mehr zwischen dem Primär- und dem Sekundärinsider. Diese Unterscheidung greift allerdings der Gesetzgeber zur Beurteilung der Strafbarkeit des verbotenen Insiderverhaltens wieder auf, wobei er die Strafbarkeit von Insidern überaus komplex gestaltet:

b) Strafbarkeit des Insider-Geschäften

Nach dem System der Straftatbestände der

1. $\S 38$ Abs. 2 i.V.m. $§ 39$ Abs. 2 Nr. 3 i.V.m. $§ 14$ Abs. 1 Nr. 2 oder

2. $\S 38$ Abs. 2 i.V.m. $\S 39$ Abs. 2 Nr. 4 i.V.m. $§ 14$ Abs. 1 Nr. 3 oder

3. $\S 38$ Abs. $1 \mathrm{WpHG}$

ist grundsätzlich jedem Insider (Primär- und Sekundärinsider) der Erwerb, oder die Veräußerung von Insiderpapieren ( 14 Abs. 1 Nr. 1 WpHG), unter Verwendung der Insider-Kenntnis verboten, während allein dem Primärinsider die Mitteilung oder das Zugänglichmachen (§ 39 Abs. 2 Nr. 3 WpHG), bzw. die Erwerbs- oder/und Veräußerungsempfehlung (das sog. Tipping) und die Verleitung zu Insider-Geschäften ${ }^{31}$ (§ 39 Abs. 2 Nr. 4 WpHG) untersagt ist.

Abbildung 2: Insiderstrafbarkeit

\begin{tabular}{|c|c|c|c|}
\hline $\begin{array}{l}\text { Bezeich- } \\
\text { nung }\end{array}$ & $\begin{array}{l}\text { Insider-Eigen- } \\
\text { schaft }\end{array}$ & $\begin{array}{l}\text { Handlungs-Verbote } \\
\text { (i.S.d. } \$ 14 \text { WpHG) }\end{array}$ & Strafe \\
\hline \multicolumn{2}{|c|}{ Für alle Insider } & $\begin{array}{l}\text { 1. Erwerb, } \\
\text { 2. Veräußerung von Insiderpa- } \\
\text { pieren ( } \$ 14 \text { Abs. } 1 \mathrm{Nr} .1) \text {, un- } \\
\text { ter Verwendung der Insider- } \\
\text { Kenntnis (Kausalität) }\end{array}$ & $\begin{array}{l}38 \text { Abs. } 1 \\
\text { Nr. } 1\end{array}$ \\
\hline $\begin{array}{l}\text { Primär- } \\
\text { Insider }\end{array}$ & $\begin{array}{l}\text { Organ-Insider } \\
\S 38 \text { Abs. } 1 \mathrm{Nr} .2 \mathrm{a} \\
\text { Beteiligungs-Insider } \\
\text { § } 38 \text { Abs. } 1 \mathrm{Nr} .2 \mathrm{~b} \\
\text { Berufs-Insider } \\
\text { § } 38 \text { Abs. } 1 \mathrm{Nr} .2 \mathrm{c} \\
\text { Straftats-Insider } \\
\S 38 \text { Abs. } 1 \mathrm{Nr} .2 \mathrm{~d}\end{array}$ & $\begin{array}{l}\text { 3. Mitteilen oder Zugänglichma- } \\
\text { chen ( } \$ 39 \text { Abs. } 2 \text { Nr. 3), } \\
\text { 4. Erwerbsempfehlung und Ver- } \\
\text { äußerungsempfehlung (»TIP- } \\
\text { PING«) } \\
\text { 5. Verleitung ( } \$ 39 \text { Abs. } 2 \text { Nr. 4) }\end{array}$ & $\begin{array}{l}\text { \$ } 38 \text { Abs. } 1 \\
\text { Nr. } 2 \text { i.V.m. } \\
\text { \$ } 39 \text { Abs. } 2 \\
\text { Nr. } 3 \text { und } 4\end{array}$ \\
\hline $\begin{array}{l}\text { Sekundär- } \\
\text { Insider } \\
\text { (Dritter) }\end{array}$ & (Kenntnis-Insider) & $\begin{array}{l}\text { 1. Erwerb, } \\
\text { 2. Veräußerung von Insiderpa- } \\
\text { pieren ( } \$ 14 \text { Abs. INr. 1), un- } \\
\text { ter Verwendung der Insider- } \\
\text { Kenntnis (Kausalität) }\end{array}$ & $\begin{array}{l}38 \mathrm{Abs} .1 \mathrm{Nr} . \\
1\end{array}$ \\
\hline
\end{tabular}

31 zu der Parallelproblematik der Anwendung des unbestimmten Tatbestandsmerkmals des $»$ Verleitens« dazu siehe oben: Kapitel I.2. (m.w.H.). 
Im Ergebnis bleibt daher dem sogen. Sekundärinsider - also demjenigen, der die Insiderinformation von einem Insider erfahren hat - lediglich die unmittelbare Verwertung dieser Information - also der Erwerb, und die Veräußerung von Insiderpapieren untersagt.

\section{c) Ordnungswidrigkeiten von Insider-Handlungen}

Die inhaltliche Unterscheidung von Strafbarkeit und Ordnungswidrigkeit traf der deutsche Gesetzgeber letztlich, um das von der Strafbarkeit ${ }^{32}$ nicht erfasste Verhalten eigens einem Bußgeldtatbestand zuzuführen und als Ordnungswidrigkeit ahnden zu können. Der neu aufgenommene Bußgeldtatbestand für Insiderverstöße ( $39 \mathrm{WpHG}$ ) nimmt nämlich unmittelbar Bezug auf den Verbotstatbestand des $\S 14$ Abs. 1, Nr. 2 und $3 \mathrm{WpHG}$ und unterzieht somit die Mitteilung oder/und das Zugänglichmachen (§ 39 Abs. 2 Nr. 3 WpHG), die Erwerbsempfehlung und die Veräußerungsempfehlung, sowie die Verleitung eines Anderen zu Erwerbs- oder Veräußerungsempfehlungen ( 39 Abs. 2 Nr. 4 WpHG) der Sanktionierung nach dem OrdnungswidrigkeitenRecht.

Abbildung 3: Ordnungswidrigkeit der Insiderhandlungen

\begin{tabular}{|c|c|c|c|}
\hline $\begin{array}{l}\text { Bezeich- } \\
\text { nung }\end{array}$ & $\begin{array}{l}\text { Insider- } \\
\text { Eigenschaft }\end{array}$ & $\begin{array}{l}\text { Handlungs-Verbote } \\
\text { (i.S.d. } \$ 14 \mathrm{WpHG} \text { ) }\end{array}$ & Buße \\
\hline \multirow[t]{2}{*}{$\begin{array}{l}\text { Alle } \\
\text { Insider }\end{array}$} & \multirow[t]{2}{*}{$\begin{array}{l}\text { Primär- und } \\
\text { Sekundärisider } \\
\text { § } 39 \text { Abs. } 2 \text { Nr. } 3\end{array}$} & $\begin{array}{l}\text { 1. Mitteilen od. Zugänglichma- } \\
\text { chen ( } \$ 39 \text { Abs. } 2 \text { Nr. 3), } \\
\text { 2. Erwerbsempfehlung und Ver- } \\
\text { äußerungsempfehlung (»TIP- } \\
\text { PING«) }\end{array}$ & $\begin{array}{l}\S 39 \text { Abs. } 2 \\
\text { Nr. } 3\end{array}$ \\
\hline & & 3. Verleitung ( $\$ 39$ Abs. 2 Nr. 4) & $\begin{array}{l}\S 39 \text { Abs. } 2 \\
\text { Nr. } 4\end{array}$ \\
\hline
\end{tabular}

Da $§ 39$ Abs. 2 WpHG den $\S 14$ Abs. 1, Nr. 1 WpHG nicht erwähnt, bleiben der Erwerb und die Veräußerung von Insiderpapieren unter Verwendung der Insiderinformation ausschließlich strafrechtlichen Sanktionen des $\S 38$ Abs. 1 Nr. 1 WpHG vorbehalten.

Die höchst differenzierte Aufteilung der Insider-Verstöße in Straf und Bußgeldvorschriften erscheint einem juristischen Laien kaum noch nachvollziehbar. Des Weiteren erfassen unterschiedliche Straf- und Bußgeldtatbestände ein und dasselbe Insider-Verhalten (hier das Mitteilen od. Zugänglichmachen (§ 39 Abs. 2 Nr. 3), die Erwerbsempfehlung und Veräußerungsempfehlung) mehrfach ${ }^{33}$. Für eine solche »doppelte« Absicherung des Gesetzgebers fehlt ein Rechtsbedürfnis.

32 Strafbarkeit nach $\S 38$ Abs. 2 i.V.m. $\S 39$ Abs. 2 Nr. 3 i.V.m. $\S 14$ Abs. 1 Nr. 2 oder, $\S \S 38$ Abs. 2 i.V.m. $\S 39$ Abs. 2 Nr. 4 i.V.m. § 14 Abs. 1 Nr. 3 oder, $\S 38$ Abs. 1 WpHG.

33 Lediglich die Sanktionierung des Erwerbs und der Veräußerung von Insiderpapieren erfolgt ausschließlich nach $\S 38$ Abs. 1 Nr. 1 WpHG. 


\section{Regelungen zur Verhinderung von Marktmanipulationen}

Lange Zeit war fraglich, inwieweit insbesondere die Fallgruppe des sog. »Scalping « strafrechtlich zu bewerten war.

Beim Scalping empfiehlt ein sog. Sachkundiger oder »Börsen-Experte« einem groBen Publikum den Erwerb einer Anlage, nur zu dem Zweck das Kaufverhalten seines Publikum anzureizen, dadurch den Kurs dieser Anlage zu steigern, und somit eigene Vorteile - etwa durch den Verkauf der nun wertmäßig gestiegenen Anlage realisieren zu können. Speziell Fernsehsendungen oder Internet-Auftritte bieten derartigen Börsen- und Anlagespezialisten bereitwillig eine effektive Plattform ihrer »Beratungstätigkeit «.

Die

- Richtlinie 2003/6/EG des Europäischen Parlaments und des Rates vom 28. Januar 2003 über die Insider-Geschäfte und Marktmanipulationen (MarktmissbrauchsRichtlinie) ${ }^{34}$

- Richtlinie 2003/124/EG der Kommission vom 22. Dezember 2003 zur Durchführung der Richtlinie 2003/6/EG des Europäischen Parlamentes und des Rates betreffend die Begriffsbestimmung und die Veröffentlichung von Insider-Informationen und die Begriffsbestimmung der Marktmanipulation ${ }^{35}$

- Richtlinie 2004/72/EG der Kommission vom, 29. April 2004 zur Durchführung der Richtlinie 2003/6/EG des Europäischen Parlaments und des Rates vom 28. Januar 2003 über die Insider-Geschäfte und Marktmanipulationen (MarktmissbrauchsRichtlinie) ${ }^{36}$

sowie die

- Verordnung (EG) Nr. 2273/2003 der Kommission vom 22. Dezember 2003 zur Durchführung der Richtlinie 2003/6/EG des Europäischen Parlaments und des Rates vom 28. Januar 2003 über die Insider-Geschäfte und Marktmanipulationen ${ }^{37}$

haben die Reglementierung bzw. das Verbot des Scalping in den nationalen Rechtsordnungen veranlasst.

Die vormalige Regelung des deutschen Rechts war ungeeignet strafrechtliche Sanktionen gegen den »Scalper« aufzubieten ${ }^{38}$. Denn die rechtliche Besonderheit des Scalping besteht darin, dass - wie vom Scalper angekündigt - die Kurse, nach der Bekanntgabe der Anlageempfehlung des Scalpers, tatsächlich steigen ${ }^{39}$. Die Information ist daher gerade nicht falsch. Ihrer strafrechtlichen Einordnung als irreführende, oder betrügerische Handlung steht daher das strenge Analogieverbot des § $1 \mathrm{StGB}$ entgegen. Auch die damalige Klarstellung in dem Vorläufer der heutigen deutschen Markt-

34 ABl. Nr. L 96 vom 14.04.2003, S. 16 ff.

35 ABl. Nr. L 339 vom 24.12.2003, S. 70 ff.

36 ABl. Nr. L 162 vom 30.04.2004, S. 70 ff

37 ABl. Nr. L 336 vom 23.12.2003, S. 33 ff.

38 Krimphove: Aktuelle Entwicklung im europäischen Bank- und Kapitalmarktrecht, in: Zeitschrift für das gesamte Kreditwesen, 2/2005, S. 35 ff. $97 \mathrm{ff}$.

39 Wenngleich auch diese Steigerung nicht wegen der Werthaltigkeit der Anlage, sondern auf der Werbung für und der dadurch eintretenden Nachfragesteigerung beruht. 
manipulations-Konkretisierungs-Verordnung (MaKonV) genügte nicht, strafrechtliche Sanktionen gegen das Scalping zu begründen. Der deutsche Gesetzgeber hat zwar im Jahr 2005 »nachgebessert«. Allerdings erreichte er damit eine inhaltlichen wie systematischen Undurchschaubarkeit der derzeitigen Rechtslage: So kommen beispielsweise zur straf- als auch zur ordnungsrechtlichen Beurteilung des »Scalping « gleich 4 Alternativen - nämlich die hier angegebenen Alternativen 1, 3 und 4 - und nur in besonders gelagerten Fällen die Alternative Nr. 2 in Betracht, deren Abgrenzung und inhaltliche Unterscheidung problematisch ist:

Das Sanktionssystem der Marktmanipulation:

1. Ordnungswidrig nach $\S 39$ Abs. 2 Nr. 11 i.V.m. $§ 20$ a Abs. 1 Nr. 1 WpHG handelt, wer vorsätzlich oder leichtfertig unrichtige oder irreführende Angaben macht, die zur Bewertung eines Finanzinstrumentes erheblich sind.

2. Ebenfalls ordnungswidrig handelt, nach $\S 39$ Abs. 1 Nr. 1 i.V.m. $§ 20$ a Abs. 1 Nr. 2 WpHG wer Geschäfte oder Kauf- bzw. Verkaufsaufträge tätigt, die geeignet sind, falsche oder irreführende Signale zusetzen oder ein künstliches Preisniveau herbeizuführen;

oder

3. wer nach $\S 39$ Abs. 1 Nr. 2 i.V.m. § 20a Abs. 1 Nr. 3 WpHG sonstige Täuschungshandlungen vornimmt, die geeignet sind, auf den Preis eines Finanzinstrumentes einzuwirken.

4. Strafbar nach $\S 38$ Abs. 2 i.V.m. § 39 Abs.- 1, Nr. 1 u. Nr. 2, oder Nr. 11, i.V.m. § 20a WpHG handelt, wer eine der oben angegebene drei Alternativen vorsätzlich begeht und dadurch auf den Preis eines Finanzinstrumentes einwirkt.

Die Gesetzessystematik erscheint ferner dadurch inhaltlich problematisch, dass der deutsche Gesetzgeber - ausdrücklich in $\S \S 39$ Abs. 1, § 39 Abs. 2 und $\S 39$ Abs. 11 $\mathrm{WpHG}$ also für alle o.g. Alternativen der Ordnungswidrigkeit und Strafbarkeit den Begriff der Täuschungshandlung aus $\S 4$ der MaKonV herleitet, obschon der Wortlaut des $\S 4$ Abs. 1 MaKonV sich nur auf die »sonstigen Täuschungshandlungen« i.S.d. $\S$ 20a Abs. 1 Satz 1 Nr. 3 WpHG bezieht.

Allein die, die Strafbarkeit des Scalpers begründende, viergliedrige Paragrafenkette - $\$ 38$ Abs. 2, i.V.m. § 39 Abs. 1, Nr. 1 und Nr. 2, oder Nr. 11, i.V.m. § 20a WpHG i.V.m § 4 MaKonV - lässt Zweifel aufkommen, inwieweit der deutsche Gesetzgeber die Klarheit und Verständlichkeit der Rechtslage im Auge hatte.

$\mathrm{Zu}$ der problematischen Strafbegründung durch Normen der Exekutive die, wie im Fall des $§ 4$ MaKonV und der zweifelhaften Vorgehensweise, europarechtliche Vorgaben in deutschen Recht mit strafbegründendem Bezug umsetzen, obschon der Europäische Gesetzgeber keinerlei Kompetenz für nationale Strafregelungen besitzt, wurde bereits oben - im Zusammenhang mit der Übernahme der Regelung des § 2 MaKonV - hingewiesen ${ }^{40}$. Dieselben Bedenken gelten auch an dieser Stelle.

40 Siehe oben: Kapitel I.1.a. 
Strafrechtlich überdenkenswert ist ebenfalls der inhaltliche Verweis der $\S \S 38$ i.V.m. § 39 Abs.1 i.V.m. § 20a WpHG auf $§ 4 \mathrm{MaKonV}^{41}$, denn dieser Verweis eröffnet eine weite Strafbarkeitsbegründung:

- § 4 Abs. 1 MaKonV definiert sonstige Täuschungshandlungen als Irreführung wobei Absatz 2 des $\S 4$ MaKonV lediglich Indizien in Form von nichtabschließenden Regelbeispielen für das Vorliegen einer Täuschungshandlung liefert.

- Zu deren Feststellung einer Täuschungshandlungen stellt $\S 4$ Abs. 1 dann auf die Sicht eines »verständigen Anlegers«ab.

- Dabei genügen schon von einem wirtschaftlichen Interesse beeinflusste Finanzanalysen oder Anlageempfehlungen, um eine Täuschungshandlung zu begründen.

- Neu ist dann, dass $§ 4$ Abs. 3 MaKonV die Kundgabe einer Stellungnahme oder eines Gerüchts über das Finanzinstrument, oder dessen Ausgeber in den Medien dann immerhin als sonstige Täuschungshandlung ausreicht, wenn der entsprechenden Interessenkonflikt nicht während der Kundgabe in angemessener Form - durch wen auch immer - offen gelegt wird.

41 Siehe oben: in diesem Kapitel. 
Abbildung 4: Sanktionssystem einer Marktmanipulation (hier: Scalping) $§ 38$ Abs. 2, i.V.m. § 39 Abs. 1, Nr. 1 und Nr. 2, oder Nr. 11, i.V.m. § 20a WpHG i.V.m § 4 MaKonV

\begin{tabular}{|c|c|c|c|c|}
\hline $\begin{array}{l}\text { Straftat- } \\
\text { bestand }\end{array}$ & \multicolumn{2}{|c|}{ Ordnungswidrigkeit } & \multicolumn{2}{|c|}{ Tathandlung } \\
\hline \multirow[t]{7}{*}{$\begin{array}{l}\S 38 \text { Abs. } 2 \\
\text { i.V.m. }\end{array}$} & $\begin{array}{l}39 \text { Abs. } 2 \\
\text { Nr. } 11 \\
\text { i.V.m. }\end{array}$ & $\begin{array}{l}\text { 20a Abs. } 1 \\
\text { Satz } 1 \\
\text { Nr. } 1\end{array}$ & \multicolumn{2}{|c|}{ 1. Unrichtige, irreführende Angaben } \\
\hline & $\begin{array}{l}39 \text { Abs. } 1 \\
\text { Nr. } 1 \text { i.V.m }\end{array}$ & $\begin{array}{l}\text { 20a Abs. } 1 \\
\text { Satz } 1 \mathrm{Nr} . \\
2\end{array}$ & \multicolumn{2}{|c|}{$\begin{array}{l}\text { 2. Geschäftsvornahme (Kauf, Veräu- } \\
\text { ßerung } v \text {. Wertpapieren) oder } \\
\text { 3. Auftragserteilung zu solchen Ge- } \\
\text { schäften }\end{array}$} \\
\hline & \multirow{5}{*}{$\begin{array}{l}39 \text { Abs. } 1 \\
\text { Nr. } 2 \text { i.V.m }\end{array}$} & \multirow{5}{*}{$\begin{array}{l}\text { 20a Abs. } 1 \\
\text { Satz } 1 \\
\text { Nr. } 3\end{array}$} & \multicolumn{2}{|c|}{ 4. Sonstige Täuschungshandlungen } \\
\hline & & & \multicolumn{2}{|c|}{$\begin{array}{l}\text { Definition sonstige Täuschungshand- } \\
\text { lungen }\end{array}$} \\
\hline & & & $\begin{array}{l}4 \text { Abs. } 1 \\
\text { MaKonV }^{43}\end{array}$ & I. Irreführung \\
\hline & & & $\begin{array}{l}4 \text { Abs. } 2 \\
\text { MaKonV }\end{array}$ & $\begin{array}{l}\text { II. Indizien für } \\
\text { »sonst. Täu- } \\
\text { schungshand- } \\
\text { lung« } \\
\text { a) Irreführungen, } \\
\text { b) von wirt- } \\
\text { schaftlichem } \\
\text { Interesse be- } \\
\text { einflusste (ob- } \\
\text { jektiv rich- } \\
\text { tige) Analysen }\end{array}$ \\
\hline & & & $\begin{array}{l}4 \text { Abs. } 3 \\
\text { MaKonV }\end{array}$ & $\begin{array}{l}\text { III.Stellung- } \\
\text { nahme in Me- } \\
\text { dien/ ohne } \\
\text { Hinweis auf } \\
\text { einen Interes- } \\
\text { senkonflikt }\end{array}$ \\
\hline
\end{tabular}

42 Verordnung zur Konkretisierung des Verbotes der Marktmanipulation (Marktmanipulations-Konkretisierungsverordnung) v. 1. 3. 2005 (BGBl. I S. 515). 
Ging die Rechtsprechung deutscher Obergerichte noch sehr großzügig von der inhaltlichen Bestimmtheit des $§ 20 \mathrm{a}$ Abs. $1 \mathrm{WpHG}$ aus ${ }^{43}$, so erscheint diese Sicht angesichts der Interpretation des $\S 20$ a Abs. 1 mit $\S 4$ Abs. 3 MaKonV mehr als fragwürdig: Was bedeutet die in $\S 4$ Abs. 3 MaKonV vorgeschriebene angemessene Offenlegung? Wie muss der offen zu legende Interessenkonflikt beschaffen sein? Genügt zur Annahme eines Interessenkonfliktes in dem sich der Täter befindet auch ein Beteiligtsein von Familienangehörigen? in welcher Form hat sie zu erfolgen? Dies alles sind Fragen die inhaltlich völlig unbeantwortet sind und damit die Strafnorm des $\S \S 38$ i.V.m. 39 Abs. 1 i.V.m. 20a WpHG i.V.m. § 4 MaKonV grundgesetzwidrig i.S.d. § 103 GG erscheinen lassen. Allein der Wortlaut des $§ 4$ Abs. 3 MaKonV mit seinen inhaltsleeren Aufzählungen ${ }^{44}$ spricht dafür, dass der deutsche Gesetzgeber einen »Auffangtatbestand « konstruieren wollte, der - in jedem Fall - das Scalping der Strafe unterstellt. Auch der Konstruktion eines »Auffangtatbestandes« stehen die grundgesetzlichen Bedenken der Unbestimmheit einer Strafnorm gegenüber ${ }^{45}$.

Zudem bewirkt die Übernahme europäischer Vorgaben in das deutsche Bank- und Wertpapierhandelsrecht - wie schon bei der Regelung von Insiderhandelsverboten ${ }^{46}$ eine »Überregulierung «: Denn systematisch ermöglicht der Verweis in unterschiedlichen Paragrafenketten auf die Regelungen des § 4 MaKonV, dass ein und dieselbe Handlungsweise (hier das Scalping) gleich sechsmal strafrechtlich relevant gewürdigt werden kann.

Strafrechtliche Beurteilungsmöglichkeiten des Scalping nach der derzeitigen Rechtslage

1. $\S 38$ Abs. 2 i.V.m. 39 Abs. 2 Nr. 11 i.V.m. 20a Abs. 1 Satz 1 Nr. 1 i.V.m. 4 Abs. $1 \mathrm{MaKonV}$

2. $\S 38$ Abs. 2 i.V.m. 39 Abs. 2 Nr. 11 i.V.m. 20a Abs. 1 Satz 1 Nr. 1 i.V.m. 4 Abs. 2 Nr. 2 MaKonV

3. $\S 38$ Abs. 2 i.V.m. 39 Abs. 2 Nr. 11 i.V.m. 20a Abs. 1 Satz 1 Nr. 1 i.V.m. 4 Abs. 3 Nr. 2 MaKonV

4. $\S 38$ Abs. 2 i.V.m. 39 Abs. 1 Nr. 2 i.V.m. 20a Abs. 1 Satz 1 Nr. 3 i.V.m. 4 Abs. $1 \mathrm{MaKonV}$

5. $\S 38$ Abs. 2 i.V.m. 39 Abs. 1 Nr. 2 i.V.m. 20a Abs. 1 Satz 1 Nr. 3 i.V.m. 4 Abs. 2 Nr. 2 MaKonV

6. $§ 38$ Abs. 2 i.V.m. 39 Abs. 1 Nr. 2 i.V.m. 20a Abs. 1 Satz 1 Nr. 3 i.V.m. 4 Abs. 3 Nr. 2 MaKonV

Die vom Gesetzgeber zur strafrechtlichen Bewertung von Scalping offenbar am geeignetsten angesehene Alternative ist Nr. $6^{47}$. Allerdings ist die Abgrenzung zwischen

43 BGH WM 2004,S. 69 ff. 73 (m.w.H.); im Einzelnen: Schröder: in: Achenbach, Ransiek (Herg): Handbuch Wirtschaftsstrafrecht, Heidelberg 2004, Kapitel X 2, Rn. 52 (m.w.H.).

44 »die Nutzung eines gelegentlichen oder regelmäßigen Zugangs zu traditionellen oder elektronischen Medien .... .

45 zur Parallelproblematik des vormaligen $\S 88$ Nr. 2 BörsG: BVerfGE 45, S. 363 ff., 371 ff. (m.w.H.).

46 Siehe oben: Kapitel: I. 3. c. (m.w.H.).

$47 \S \S 38$ Abs. 2 i.V.m. 39 Abs. 1 Nr. 2 i.V.m. 20a Abs. 1 Satz 1 Nr. 3 i.V.m. 4 Abs. 3 Nr. 2 MaKonV. 
den Alternativen 1 - 6 zwingend erforderlich, enthält doch nur sie Möglichkeit der »Exkulpation« durch den Hinweis auf einen bestehenden Interessenkonflikt«.

Die Wiedergabe der aktuellen Rechtslage verdeutlicht, dass die Sanktionierung von »Scalping « - auch nach der Einführung des europäischen Rechts - keineswegs gelungen ist.

\section{Zusammenfassung und Resümee}

Der deutsche Gesetzgeber hat europäisches Recht - insbesondere wenn es sich um europäische Richtlinien handelt - in sein nationales Recht zu überführen. Dabei stößt der Gesetzgeber gerade in Rechtsgebieten in denen das umzusetzende europäische Recht auf eine bereits detailliert entwickelte nationale Systematik und/oder Dogmatik trifft auf erhebliche Umsetzungsprobleme.

Diese Umsetzungsprobleme hat der deutsche Gesetzgeber nicht bewältigt. In der Praxis zeigt sich vielmehr, dass er sie dadurch umgeht, dass er die Vorgaben das Europäische Recht zu dem bestehenden nationalen einfach »addiert«. Hieraus entstehen aufgeblähte und unüberblickbare nationale Gesetzeskonstruktionen. Ihr Mangel an intellektueller Durchdringbarkeit dieser Konstruktionen wäre nicht dass größte Problem. Von grundsätzlicher Bedeutung ist, dass das Verfassungsrecht und das Strafrecht die inhaltliche Bestimmtheit von Straftatbeständen zwingend vorschreiben. Gerade hinter dem Gebot, nicht nur eine verständliche, sondern auch eine inhaltlich klare, bestimmte und nachvollziehbare Regelung zu schaffen bleibt der deutsche Gesetzgeber zurück.

$\mathrm{Zu}$ einem weiteren europa- und verfassungsrechtlichen Problem führt die Bereitschaft des deutschen Gesetzgebers, europäische Vorgaben in deutsches Strafrecht dadurch umzusetzen, dass er den Erlass von »Klärungsvorschriften« wie etwa bei der »Verordnung zur Konkretisierung des Verbotes der Marktmanipulation v. 1. 3. 2005« der Exekutive überlässt. Die Exekutive konkretisiert nämlich dann europäische Normen für ein Rechtsgebiet, für dessen Regelung der Europäische Gesetzgeber keinerlei Kompetenz hat.

Mit zukünftig zunehmender Regelungsintensität des europäischen Gesetzgebers auf dem Gebiet des Bank- und Wertpapierhandelsrechts steigt die Bedeutung der hier vorgestellten Problematik.

Zugegebenermaßen hat der deutsche Gesetzgeber aktuell noch andere »Baustellen « der Harmonisierung des deutschen und europäischen Rechts zu bearbeiten (insbesondere: das Zertifikatrecht und Ratingrecht, sowie zahlreiche prospektrechtliche Fragen $^{48}$ ), und zugegebenermaßen zählt das deutsche Bank- und Wertpapierhandelsrecht eben nicht $\mathrm{zu}$ den systematisch/dogmatisch anspruchslosesten und gradlinigsten Rechtsbereichen, in denen der deutsche Gesetzgeber seinen Harmonisierungsbemü-

48 Krimphove: Rechts- und Haftungsfragen des externen Unternehmensratings, in: Finanz Betrieb Mai 2005, S. 333 ff., Krimphove: Zertifikathandel in Deutschland, In: Finanz Betrieb, 2006, S. 255 ff.; Krimphove: Bestandsaufnahme und Ausblick auf ein neu zu schaffendes Ratingrecht, in: Achleitner/Everling (Hrsg.): Rechtsfragen im Rating, S. 65 ff. (m.w.H.) Wiesbaden 2005. 
hungen ohne weitere Sorgfalt nachgehen kann. Dennoch verdeutlicht gerade die oben dargestellte strafrechtliche Problematik des deutsch/europäischen Bank- und Wertpapierhandelsrechts dass Fortsetzung der bisherigen deutschen Praxis europarechtliche Normen in sein Gesetz umzusetzen unbrauchbar ist.

Hier ist der deutsche Gesetzgeber bereits heute aufgefordert eine neue systematisch, schlüssige verfassungs- wie europarechtskonforme Gesetzeslage zu erarbeiten und vorzulegen. 